\title{
Tourisme, risques sanitaires et enjeux médiatiques : psychose autour d'Ebola au Sénégal
}

Tourism, health hazards and media issues: psychosis around ebola in Senegal Turismo, rischi per la salute e le questioni del media : la psicosi intorno ebola in Senegal

\section{Adama Ndiaye et Moussa Mbow}

\section{(2) OpenEdition}

\section{Journals}

\section{Édition électronique}

URL : http://journals.openedition.org/ctd/932

DOI : $10.4000 /$ ctd. 932

ISSN : 2491-1437

\section{Éditeur}

Chaire Unesco Pratiques émergentes en technologies et communication pour le développement

\section{Édition imprimée}

Date de publication : 30 octobre 2016

ISBN : 2491-1437

\section{Référence électronique}

Adama Ndiaye et Moussa Mbow, «Tourisme, risques sanitaires et enjeux médiatiques : psychose autour d'Ebola au Sénégal », Communication, technologies et développement [En ligne], 3 | 2016, mis en ligne le 10 octobre 2016, consulté le 13 juin 2020. URL : http://journals.openedition.org/ctd/932 ; DOI : https://doi.org/10.4000/ctd.932

Ce document a été généré automatiquement le 13 juin 2020.

Communication, technologies et développement 


\section{Tourisme, risques sanitaires et enjeux médiatiques : psychose autour d'Ebola au Sénégal}

Tourism, health hazards and media issues : psychosis around ebola in Senegal Turismo, rischi per la salute e le questioni del media : la psicosi intorno ebola in Senegal

\section{Adama Ndiaye et Moussa Mbow}

1 Le tourisme sénégalais traverse une crise longue que certains qualifient même de structurelle aujourd'hui. De 1998 à nos jours, seules les années 2004 à 2007 ont connu des pics d'espoir. L'année 2012 (980 000 touristes ont généré 352,6 milliards de F CFA de recettes ${ }^{1}$ ), qui avait beaucoup promis, a été compromise par les péripéties électorales précédées des émeutes de l'électricité dans les banlieues dakaroises et du mouvement du 23 juin 2011 suite au refus public d'une révision constitutionnelle. Le Sénégal sort de cette ornière politique avec l'espoir de relance en 2013 d'un des secteurs clés, le tourisme, qui se classe au second rang de son économie avec une contribution au PIB de l'ordre de 6,7\%. Mais c'était sans compter en 2014 avec le désastre d'une rumeur médiatique d'Ebola ${ }^{2}$.

2 Comment dans un tel contexte imaginer un futur touristique pour une activité déjà en sursis? Que révèle cette psychose d'Ebola au Sénégal, dans son approche communicationnelle? Et quel est l'impact de cette intrusion d'Ebola et de sa médiatisation sur le tourisme?

3 Les réponses que nous apportons à ces questionnements d'ordre sociologique et économique se feront à travers une lecture d'ouvrages dédiés au risque, à l'économie touristique et aux médias dans leur diversité : presse écrite, audiovisuelle, internet et médias, sites web et réseaux sociaux. Toutefois, il est important de rappeler que le traitement médiatique de cette question d'Ebola n'est pas que social ou sanitaire. Pour le Sénégal, la conséquence économique de la surmédiatisation de l'épidémie a provoqué 
un marasme touristique inquiétant. La psychose créée autour de cette maladie a sapé toutes les tentatives de relance de cette seconde mamelle de l'économie nationale.

Dans cette étude, nous mettons l'accent sur deux axes majeurs. D'abord, le risque sanitaire et ses enjeux médiatiques. Dans cette partie nous tentons de présenter une revue d'écrits consacrés au risque sanitaire avant de faire l'analyse du traitement médiatique d'une maladie à panique. Nous traitons ensuite l'effet psychose du risque d'Ebola et ses impacts touristiques...

\section{Gestion du traitement médiatique d’Ebola au Sénégal}

\section{Amplification, représentation et perception du risque}

5 Décrit comme une épidémie, pour son effet psychose surtout, Ebola se présente d'après l'Organisation mondiale de la santé (OMS) comme une maladie tropicale infectieuse causée par un virus. Les symptômes principaux sont la fièvre et les hémorragies. Dans la plupart des cas, la maladie aboutit au décès du patient. Elle s'est déclarée pour la première fois en 1976 près du fleuve Ebola (en République Démocratique du Congo, ancien Zaïre). Depuis, la maladie survient de manière récurrente dans différents pays africains, sous forme de petites épidémies. Ebola se transmet d'homme à homme. A ce jour, on ignore toujours quel en est le véritable vecteur. Les singes et d'autres animaux hôtes sont mis en cause dans la propagation du virus. Les humains contaminés, mais en bonne santé, sont également suspectés de transmettre la maladie. Il n'existe encore aucun vaccin contre Ebola qui demeure une maladie à déclaration obligatoire.

6 La dernière épidémie est probablement celle qui a été la plus meurtrière ; elle a été la première diagnostiquée hors d'Afrique centrale et a touché principalement trois pays de l'Afrique de l'Ouest (Guinée, Sierra Leone, Liberia). D'autres pays ont connu des cas, mais avec des proportions moins alarmantes. L'épidémie a commencé en décembre 2013 en Guinée avant de gagner la Sierra Leone et le Liberia, respectivement en mars et mai 2014. Elle y a causé beaucoup de victimes. Elle fait son entrée au Sénégal le 29 septembre 2014 avec un cas venu de la Guinée. Peut-on parler de «risque » pour ce pays?

«On peut dire qu'il y a risque, lorsque qu'il y a danger, menace, crainte d'un péril quelconque. L'imminence du danger est donc caractéristique d'un état à risque. Certaines considérations populaires du risque appréhendent souvent ce phénomène en fonction de catastrophes naturelles. Néanmoins, des auteurs soulignent sa complexité en mettant en avant les notions de vulnérabilité et de dommage $»^{3}$.

7 Le risque est le produit de l'aléa (probabilité d'occurrence d'un phénomène naturel ou technologique) et de la vulnérabilité (la sensibilité du lieu, sa propension à subir des dommages) ; la vulnérabilité s'estime également en fonction des capacités de réaction de la société. La notion de risque est donc complexe et fluctuante dans le temps comme dans l'espace. L'aléa et la vulnérabilité n'étant jamais des données stables.

8 L'information à risque pourrait être assimilée à la diffusion de nouvelles aux impacts préjudiciables. Ce sont donc des informations qui comportent en elles-mêmes toutes les caractéristiques d'un aléa provoquant ou instaurant une situation de danger et d'insécurité. Cependant, l'information à risque pourrait aussi être d'une certaine ambivalence. D'une part, elle peut être synonyme de dangereuses nouvelles, préjudiciables. D'autre part, l'information peut se traduire par une mesure de 
prévention. La diffusion d'une information à risque invoque la prise de précautions et le déploiement de mesures ou de stratégies de gestion de crise. C'est dire l'importance des médias, "acteurs incontournables de la perception du risque " (Gwinner, 2012, p. 72) ; les mass-médias contribuent d'abord à la connaissance du danger, ensuite à sa prise en compte dans l'agenda de l'opinion publique et des autorités compétentes.

«Le premier contact de l'homme de la rue avec un danger potentiel passe via les informations médiatiques. Il s'agit d'un savoir d'experts - souvent celui de scientifiques rompus à ce genre de risque - qui est proposé. En fait, les médias ont un rôle crucial dans la transformation d'un savoir d'experts en savoir de sens commun. Les informations ne présentent pas une "photocopie" du savoir savant sur le risque. Elles simplifient et rendent l'événement exceptionnel, créant des débats autour de la responsabilité et de la possibilité de blâmer dans l'espoir d'attirer l'attention de l'opinion ». (Joffe, 2005, p. 125)

Grâce aux médias, l'information est donc rendue accessible au plus grand nombre à travers cette "transformation du savoir d'experts en savoir de sens commun » avec toutes les conséquences que cela peut engendrer. Le processus débouche alors sur trois éléments : l'amplification, la représentation et la perception du risque.

Le premier élément est lié à la diffusion accélérée et abondante de l'information grâce aux médias traditionnels (presse écrite, radio et télévision) et aux nouveaux médias (internet et réseaux sociaux). Sur ce plan, il est intéressant de souligner la surenchère et l'emballement médiatique provoqués par Ebola, aussi bien en Afrique qu'ailleurs dans le monde. En un temps record, sa médiatisation a fait que cette épidémie a relégué au second plan d'autres pandémies toutes aussi importantes voire plus meurtrières.

11 Avec la représentation et la perception, on s'intéresse au contenu et à la réception des informations concernant le risque en question. S'il est vrai que la récurrence du sujet constitue un élément d'appréciation par rapport à sa représentation et sa perception, la façon dont il est abordé et traité par les médias reste le paramètre le plus essentiel. On peut penser à sa « dramatisation avec des scénarios possibles » (Joffe 2005, p. 123), ou à « des références à d'autres épidémies qui ont marqué l'Histoire par leur nombre record de victimes » (Gwinner 2012, p. 73).

\section{Le traitement médiatique d'Ebola au Sénégal}

12 Les discours véhiculés par les médias peuvent alimenter la peur et contribuer à installer la psychose et des inquiétudes au niveau des populations. Concernant Ebola, notre analyse va principalement porter sur deux quotidiens, Le Soleil et L'Observateur (tableau 1). Evidemment, le corpus ne se limite pas seulement à ces deux titres, d'autres supports médiatiques ont également été consultés ${ }^{4}$. Trois critères d'évaluation ont été retenus : le volume d'articles consacrés à la maladie, les angles de traitement et les sources de l'information, la dramatisation de l'événement à travers les registres discursifs.

Tableau $n^{\circ} 1$ : Articles consacrés à Ebola dans deux quotidiens sénégalais du 29 août au 20 septembre 2014

\begin{tabular}{|l|l|l|l|}
\hline \multicolumn{2}{|l|}{ Articles du Soleil sur Ebola } & \multicolumn{2}{l|}{ Articles de L'Observateur sur Ebola } \\
\hline Rubriques & Nombre d'articles publiés & Rubriques & Nombre d'articles publiés \\
\hline
\end{tabular}




\begin{tabular}{|l|l|l|l|}
\hline Actualités & 41 & Index & 13 \\
\hline Société & 2 & Actualités & 13 \\
\hline Economie & 2 & Société & 6 \\
\hline Politique et Institutions & 2 & Entretien & 1 \\
\hline Total & 47 & Spéciale & 1 \\
\hline & & Contributions & 1 \\
\hline & & Total & 35 \\
\hline
\end{tabular}

13 Une référence à la théorie Agenda setting de Maxwell Mc Combs et Donald Shaw comme grille d'analyse permet d'avancer quelques conclusions sur l'importance accordée à Ebola par les médias sénégalais. Pour rappel, les deux chercheurs américains pensent que les médias ne dictent pas la pensée des gens; par contre ils indiquent les sujets auxquels les citoyens doivent penser. En choisissant de traiter certains événements plus que d'autres, en hiérarchisant les sujets par ordre d'importance, ils mettraient en exergue certains messages diffusés de sorte à créer un débat là où ils le souhaitent. Ainsi, le nombre important d'articles et la place qui leur est réservée au sein de nos deux journaux (tableau 1) fait d'Ebola la priorité du moment pour les journalistes et par conséquent pour les populations. Par ailleurs, on remarque que le virus va progressivement s'infiltrer dans les colonnes des deux quotidiens en s'insérant dans des rubriques autres que celle réservée à l'actualité. Ebola était devenu omniprésent et inévitable parce que visible partout dans l'espace rédactionnel des médias.

14 En ayant révélé elles-mêmes le premier cas d'Ebola au Sénégal, les autorités ont (en partie) gagné la bataille de la communication. Elles se sont ainsi positionnées comme la principale source d'information des journalistes et ont tenté de les maîtriser avec des communiqués et des conférences de presse. Ceux-ci n'apportent en réalité pas beaucoup d'éléments nouveaux sur l'état de santé du jeune Guinéen malade sur le sol sénégalais. Mais ils ont permis de déployer toute une stratégie pour éviter la psychose, la peur et l'inquiétude des populations. Eugène Kaly, journaliste « spécialiste santé » du quotidien gouvernemental reconnaît la place centrale des autorités dans la gestion de cette affaire : "Elles ont vraiment bien communiqué, il y avait une bonne connexion avec les différentes structures qui ont été mises sur pied pour distiller les informations. "

C'est ainsi que les expressions «cas importé », "malade guinéen » ont été régulièrement utilisées; elles seront reprises et vulgarisées par la presse en général et nos deux titres en particulier. On se veut rassurant, et pour ce faire les journalistes doivent être briefés pour connaître et utiliser les mots appropriés. Considérés comme des «partenaires privilégiés dans la guerre contre Ebola» les acteurs médiatiques ont été invités à la retenue, au sens des responsabilités et à ne pas répandre les rumeurs. " Il fallait informer de manière responsable, c'est-à-dire permettre aux populations d'être au courant des risques liés à la maladie sans alarmer, ni installer la panique » pense le journaliste du quotidien gouvernemental. 
Lacommunicationestégalementorientéeverslechangementd'attitudesetdecomportements. Il faut souligner que de la révélation à la guérison du malade, les autorités ont fait l'agenda des acteurs médiatiques en leur indiquant d'une certaine manière la voie à suivre. C'est d'ailleurs la raison pour laquelle les médias ont été un levier sur lequel elles se sont appuyées pour un appel au calme et une meilleure sensibilisation des populations sur les gestes qui sauvent.

De leur côté, les médias sénégalais ont certainement été influencés par l'affaire de $L a$ Tribune en ayant traité l'événement avec plus de retenue et de responsabilité. Quelques jours avant l'apparition du premier cas, ce journal avait défrayé la chronique en révélant à sa Une « cinq cas d'Ebola au Sénégal ». Dans un communiqué, les autorités démentaient une information pouvant mettre «le pays en quarantaine de la communauté internationale » avec des conséquences sur la mobilité des Sénégalais. Après jugement, le patron de La Tribune écope d'une peine d'un an d'emprisonnement avec sursis, assortie d'une forte amende financière. C'est certainement une des raisons qui ont amené les autorités à prendre les devants avec cette vigilance vis-à-vis des acteurs médiatiques pour la gestion $\mathrm{du}$ « cas importé ».

18 Comme pour tenter de mettre fin à la psychose, la télévision publique (RTS1) montre le 19 septembre 2014 le Guinéen guéri : « il a été sauvé de la mort » clament les autorités. Mis en quarantaine et soigné à l'hôpital Fann, il a eu la chance de s'en sortir. Il se prépare alors à rentrer dans son pays. Mais avant, il dit toute sa satisfaction et parle de ses projets. Cette version de la communication gouvernementale a aussi une connotation spectaculaire. Elle est du même ressort que la communication touristique et d'entreprise. L'information est remodelée pour un but précis, c'est cette communication qu'Alain Minc (Ibid., pp. 121-122) qualifie "d'impact stimulus » aux " effets chics». Elle est présentée sous une forme plus douce, rassurante et mieux consommable. Mais ce n'est pas pour autant que les esprits du côté du tourisme vont se calmer. L'information sur ce cas Ebola au Sénégal a fait le tour du monde. Sur internet, elle est étayée et même amplifiée par les réseaux sociaux. Du coup, le cercle des « journaleux » s'élargit avec l'entrée en scène des blogueurs.

\section{Ebola dans la blogosphère}

19 La réaction des réseaux sociaux ne s'est pas fait attendre. A la suite de l'annonce officielle du premier cas d'Ebola par le ministre de la Santé Eva Marie Coll Seck du 29 août 2014, la mobilisation contre l'épidémie s'activait déjà. C'est ainsi qu'une quinzaine de jours après, le site web de Radio France Internationale (24 décembre 2015) publiait :

Au Sénégal, la lutte contre le virus Ebola s'organise sur les réseaux sociaux. Un collectif de jeunes bloggeurs a créé une page Facebook intitulée Sen Stop Ebola pour mener des campagnes de prévention auprès des populations. Internautes et personnalités soutiennent l'initiative.

Les conséquences d'une telle nouvelle sur l'économie sénégalaise étaient bien mesurées par les autorités. C'est ainsi que, selon ce site de RFI, Youssou Ndour, le célèbre chanteur sénégalais, ancien ministre de la Culture et du Tourisme, encore ministre conseiller en charge de la promotion, réagissait sur Twitter pour saluer l'engagement des bloggeurs dans cette offensive contre la menace Ebola : «Je reste admiratif devant le travail du réseau des bloggeurs du Sénégal ». 
21 Il se créait alors un réseau de bloggeurs sénégalais engagés auprès d'autres confrères africains nommés "Afriktiviste» et d'autres "influenceurs", terme utilisé dans le jargon, pour mettre en place une campagne d'information et de prévention de l'épidémie, en utilisant les réseaux sociaux. L'initiateur sénégalais de ce mouvement, Charles Vieira Sanches affirmait :

L'originalité de ce hachtag est que dès le départ, on a essayé de créer une identité forte pour que ceux qui adhèrent à cette campagne puissent transmettre aussi ce message. D'abord, dire halte à la panique, et respecter les mesures d'hygiène. À côté de l'épidémie Ebola, il y avait toute une psychose qui était une autre forme d'épidémie, qui était en train de naître. Nous voulions mettre fin à cela et relayer la bonne information. On a été contacté par un certain nombre d'activistes guinéens pour voir comment on peut les aider, maintenir cette flamme de la solidarité africaine et dire "non" à l'isolement. Il faut maintenir le cap de la prévention et celui de la solidarité. Ce n'est que comme cela qu'on réussira à bouter l'épidémie Ebola hors de la région.

Il n'en reste pas moins que la psychose nationale comme internationale n'a pu être totalement évitée.

\section{Psychose du risque d'Ebola et impacts touristiques au Sénégal}

La contre-offensive s'est activée, surtout autour de la communication sociale. Toutefois, la règle est restée simple: ne rien cacher aux médias, ce qui relève d'une approche transparente du traitement de l'information et mieux, associer toutes les parties à la gestion de cette crise. Seul cet enjeu de transparence communicationnelle et non la censure autorise, ici, d'invoquer Thierry Libaert (2010, p. 80) qui pense que «le poids des tensions allié à la logique médiatique entraîne rapidement une recherche de responsabilité. Sans se positionner en coupable, il apparaît nécessaire de fournir un acte de reconnaissance ».

Cette notion de reconnaissance renvoie à l'esprit de responsabilité, sous tendue par l'éthique et le bon sens. Cette nouvelle dimension interpelle à la fois l'informateur (souvent journaliste), mais aussi l'autorité ou le responsable d'entreprise. La responsabilité est fondamentale dans le traitement de l'information à risque ; elle l'est pour la gestion d'une crise dans une entité touristique sommée de répondre à des questions pendant et après. La crédibilité du journaliste et l'image de l'institution en dépendent. Cependant, pour l'entreprise touristique, la gestion de l'information est aussi un enjeu de survie.

L'informationestunedonnéecapablededéstabiliserlabonnemarched'uneactivitétouristique, surtout quand elle ressort de la propagande ou d'une stratégie politique commerciale que peut nourrir parfois la concurrence. On peut alors parler « d'intox ». Mais elle peut également être de source digne de foi, comme c'est le cas souvent lors d'hostilités politiques ou de crise sociale nécessitant la gestion d'une communication de crise à l'instar de cette entrée d'Ebola au Sénégal.

\section{Psychose du risque d'Ebola}

La psychose n'est rien d'autre qu'un état de panique, de crainte ou de phobie, causé par un aléa, en l'occurrence, ici, par la rumeur d'Ebola. Dans le contexte touristique du 
Sénégal, le traitement médiatique de cette épidémie s'est répandu par les réseaux sociaux. Outre la presse classique, ce sont les missions diplomatiques étrangères qui ont amplifié la donne. Un communiqué du site de l'ambassade de France (www.ambafrancesn.org/Ebola, 22 janvier 2016) évoque encore :

Compte-tenu de l'évolution de l'épidémie et de la situation des systèmes de santé au Liberia et en Sierra Leone, les pouvoirs publics recommandent aux Français résidents dont la présence n'est pas indispensable de quitter ces pays ou de surseoir à leur retour de vacances. Ils recommandent à la société Air France de suspendre temporairement sa desserte de Freetown.

De telles informations, préventives et normales, sont aussi de nature à amplifier la psychose. Or, comme nous le savons, tout traitement de l'information qui touche à la sécurité physique et morale des personnes a une incidence négative sur l'activité touristique d'un pays. Et c'est pourquoi on parle d'information à risque touristique. C'est une nouvelle dont l'écho peut réellement porter atteinte à l'économie et, au-delà, menacer l'équilibre social des populations qui vivent des retombées. Les "signaux du risque » ont été perceptibles dans le traitement médiatique du premier cas d'Ebola. On constate un champ sémantique récurrent avec l'utilisation à profusion de certains mots et expressions comme "peur ", "panique ", "psychose», " inquiétude", "stigmatisation", "calamité nationale ", "fléau », "guerre contre Ebola », « crise »...

Même si, dans la plupart des cas, on cherche soit à rassurer ou à calmer les populations, soit à alerter les autorités, l'effet contraire n'est pas exclu dès lors que ce discours phobique est mis en évidence et répété à longueur de colonnes. La peur suscitée par la maladie est-elle réelle ou supposée ? En tout cas, on note une amplification de la conscience du risque à travers certains écrits. "Ebola, cette maladie qui ne cesse de faire monter la peur chez les populations " serait sur «toutes les lèvres » à en croire L'Observateur. Le journal a également relayé des «fausses alertes » pouvant accroître le sentiment de peur. Dans le premier cas, il s'agit d'un voyageur "vomissant du sang ", suspecté d'avoir contracté le virus. Abandonné à lui-même, il mourra sans assistance, car face à la scène "c'est le sauve-qui-peut». Le deuxième cas concerne un jeune habitant de Yeumbeul (banlieue dakaroise), mort après avoir présenté les mêmes symptômes. «Du sang, vous avez dit du sang, ont interrogé les populations du quartier » rapporte le quotidien qui ajoute que le jeune homme « n'est pas mort d'E... N'empêche de la panique, il y en a eu hier à Yeumbeul Sud ». Il n'y a pas de rumeurs, un cas révélé, de la psychose et de la panique qui s'installent vont exacerber les populations.

30 En effet, des quartiers populaires de la banlieue dakaroise aux zones les plus reculées du pays, le traitement médiatique d'Ebola (à travers radios, télévision et réseaux divers), plonge les populations dans l'inquiétude, profondément marquées par la nouvelle donne. Le gel antiseptique serait introuvable à Dakar, car « les pharmacies et officines ont été prises d'assaut » dès l'annonce du premier cas d'Ebola. Quelques jours plus tard, le liquide précieux est de nouveau disponible, mais les flacons « s'arrachent comme des petits pains ». Un détour du côté de la frontière sud du pays permet de mesurer les impacts causés sur le marché hebdomadaire de Diaobé. Ici, «toutes les activités socio-économiques tournent au ralenti

31 " à cause de la fermeture des frontières annoncée le 22 août 2014. Ironie du sort, le Sénégal va se retrouver dans la même situation. Avec son « cas importé » d'Ebola, ses 
ressortissants se voient interdits d'entrer au Cameroun tandis que Banjul met en place, au niveau de la frontière sénégalaise, un dispositif de contrôle. La méfiance vis-à-vis des populations guinéennes est réelle. La stigmatisation se peaufine et il n'y a que la communication pour freiner ce parfum xénophobe.

\section{Impacts touristiques au Sénégal}

L'adage petite cause grands effets reflète l'impact d'Ebola au Sénégal. La stupeur subordonnée à ce que les acteurs du tourisme qualifient de "cacophonie médiatique ", a eu raison d'une reprise d'activités longtemps désirée. En effet, la panique, c'est ce qui a hanté tout le monde et malgré la mobilisation citoyenne d'internautes et d'autorités étatiques, l'effet médiatique d'Ebola est désastreux pour l'économie touristique. Hervé Ducruet (www.pagtour.net, 29 janvier 2015) dans son article intitulé «Le tourisme sénégalais malade d'Ebola, mais pas seulement », dit :

Le FMI explique, «Bien qu'un cas d'Ebola ait été détecté au Sénégal, ce patient a été pris en charge et l'impact sur l'économie du pays a été minime. En revanche, les conséquences d'Ebola sur le tourisme pourraient réduire le PIB annuel de $1 \% \%$. Et la situation devient alarmante. Selon les dernières statistiques publiées, le pays, qui accueillait plus d'un million de touristes en 2000, n'en reçoit plus que 450000 aujourd'hui. En crise depuis plusieurs années, le virus Ebola vient plomber encore plus l'attractivité du Sénégal.

Les crises successives quiontmarqué le tourisme sénégalais venaientd'atteindre leur paroxysme par cet effet d'Ebola, et Hervé Ducruet (Idem) rapporte :

Et s'il n'y avait qu'Ebola. «Le Quai d'Orsay avait classé le Sénégal en zone rouge, au même titre que le Mali par exemple, avant de rectifier le tir il y a une semaine. Au Sénégal, il n'y a ni Ebola, ni jihadistes, mais les gens font l'amalgame. Le secteur touristique allait déjà mal depuis plusieurs mois, mais les craintes suscitées par Ebola et le jihadistes ont porté le coup de grâce ", explique Lionel Lopez, un français propriétaire d'un hôtel dans le Sine Saloum.

En effet, les hôteliers ont aussi paniqué, car la désertion de la destination en a inquiété plus d'un: plus un seul tour opérateur ne souhaite desservir le Sénégal. Les grands hôtels sont touchés et le secteur est dans une situation d'état d'urgence. Le site d'information dakaractu. com révèle ainsi le contenu d'une conférence de presse convoquée précipitamment pour mesurer l'ampleur. Il titre: «Ebola frappe l'hôtellerie: King Fahd perd 670 millions et Terrou-bi 200 millions ». Selon ce site d'actualités,

Le succès éclatant obtenu avec la guérison de l'étudiant guinéen atteint de la fièvre hémorragique d'Ebola, Mamadou Aliou Diallo, n'a pas permis de dissiper la psychose chez les touristes. L'impact du virus Ebola au Sénégal, précisément dans le secteur touristique qui peine à atteindre sa vitesse de croisière, est alarmant. La maladie a déjà causé d'énormes dégâts sur les hôtels du pays, occasionnant une perte de plusieurs millions de francs Cfa.

L'heure est grave dit-on, et, c'est dans ce contexte de cafouillage et d'incertitude que le quotidien L'As (13 octobre 2014) annonce une réunion d'urgence de crise avec la tutelle :

Conscients que les choses s'empirent jour après jour malgré l'exploit de notre pays qui a réussi à circonscrire la maladie, les acteurs ont décidé de prendre le problème à bras le corps. Ainsi, aujourd'hui les hôteliers conduits par Mamadou Racine Sy vont se retrouver avec le ministre du Tourisme Abdoulaye Diouf Sarr. L'objectif, diton, est la mise en place d'un plan de communication pour inverser la tendance et stopper l'hémorragie. 
Dans la même veine, l'économiste Moubarak Lo, Conseiller du Président de la République (Leral.net, 1er septembre 2014) affirme :

Le risque économique de cette maladie Ebola est réel, le virus Ebola ne se contente pas de tuer des milliers de personnes, il s'emploie également à miner l'économie des pays affectés... Pour le tourisme le risque est là, car Ebola agit sur l'investissement, les ressources (rapatriement de personnes par exemple) et aussi et surtout sur l'image d'une zone, d'un pays ce qui est redoutable pour le tourisme.

En tout état de cause, la nature sensible du tourisme appelle à plus de discernement dans le traitement de l'information. C'est pourquoi il semble de bon augure pour le tourisme et pour les communautés réceptives, que l'information sur le tourisme requière de la responsabilité. Ce qui nécessite non seulement de la pédagogie, mais aussi, une véritable conscience éthique et de culture touristique. Le devoir du journalisme est d'informer vrai, mais la responsabilité du citoyen est aussi de ne pas nuire à sa communauté ou de ne pas porter atteinte aux intérêts de sa société. Selon Jean-Marie Charon (2007, p. 58) cela relève du professionnalisme, car :

Dans une démocratie les journalistes constituent une profession à haute responsabilité. La loi ne saurait tracer que quelques lignes jaunes à ne pas franchir en aucun cas. Il reste donc à chacun une large marge d'appréciation concernant leur manière de travailler et les termes dans lesquels ils doivent traiter une information. Ces règles internes à la profession constituent la déontologie (...) Audelà de la déontologie, les journalistes ont à s'interroger sur les questions qu'ils abordent et les répercussions de ce qu'ils publient et diffusent. Il s'agit là d'une réflexion d'éthique professionnelle, c'est-à-dire de morale.

On ne saurait parler de «morale » dans le jargon journalistique, mais de principe de liberté d'opinion et d'action qui sont sacrés et protégés au prix de dépénaliser le délit de presse, même si les principes de précaution et de bon sens sont inhérents à la pratique du métier. Au Sénégal, la profession elle-même peine à s'organiser.

La réglementation des médias et du corps journalistique que réclament les professionnels de ce secteur participera, sans aucun doute, à une meilleure visibilité du tourisme sénégalais. Une bonne formation du journaliste signifie ainsi une meilleure disposition au respect de la déontologie. Néanmoins, l'actualité d'Ebola au Sénégal, même si elle a contribué à aggraver la crise touristique nationale, a toutefois permis aux autorités politiques en particulier de prendre conscience de l'important enjeu économique et socioculturel du tourisme. A cet effet, en tournée économique dans la région touristique du sud, en Casamance du 19 au 25 février 2015, le Président de la République constate l'ampleur de la crise et pour démontrer sa volonté à relancer le secteur, il annonce des mesures incitatives. La Casamance bénéficie désormais du statut de zone touristique spéciale tandis que les taxes aéroportuaires ont baissé de moitié et l'encombrant visa que décriaient les acteurs depuis son application en juillet 2013 a disparu en mai 2015. N'eut été donc les effets médiatiques de cette épidémie d'Ebola aux conséquences désastreuses, cette activité touristique mourait à petit feu, bien loin du secours de l'État.

On peut retenir de cette étude une redéfinition du risque qui se présente comme un condensé de psychose et de panique qu'alimente une rumeur, médiatique dans ce casci. La chute libre de l'activité touristique sénégalaise est surtout une résultante de ces deux effets combinés. Mais en réalité le traitement médiatique de cette question d'Ebola a accentué la crise touristique qui a engagé ainsi la responsabilité du journaliste et de l'informateur en général. Il s'agit évidemment de parler du professionnalisme du 
journaliste qui est donc un enjeu de taille pour le tourisme et l'économie qu'il draine ; il peut permettre d'éviter la diffusion de fausses nouvelles dont le démenti ne réduit en rien l'ampleur, mais peut aussi et surtout aider les décideurs par le biais d'une information crédible, à prendre conscience et à réorienter judicieusement leur politique.

\section{BIBLIOGRAPHIE}

Amabert Malberti René, 2003, Autour de la mesure du risque : un questionnement multidisciplinaire, Maison des Sciences de l'Homme-Alpes.

Atenga Thomas, 2015, Epidémie d'Ebola en Afrique de l'Ouest Approches ethno-sociales comparées, Département de communication, FLSH, Université de Douala, Cameroun

Boyer Henri, 2003, Del'autrecôtédudiscours. Recherchessurlesreprésentationscommunautaires,

L'Harmattan, Paris

Carayol Valérie, Gramaccia Gino \& al, 2001, La communication du risque, Communication \& Organisation GRECO/ISIC, Université Michel de Montaigne Bordeaux 3, Talence, 2e semestre Charon Jean-Marie, 2007, Le journalisme (nouvelle édition), Les Essentiels Milan, Toulouse CREIS/ GERSULP, juin 1998, Actes du colloque, Informatisation et Anticipations, Strasbourg

Dauphiné André, 2001, Risques et catastrophes, Armand Colin, Paris De Bartillat Laurent, Retallak Simon, 2003, STOP, Seuil, Paris

Dubois Maury, 2005, Les risques naturels et technologiques, Problèmes Politiques et Sociaux

$\mathrm{n}^{\circ}$ 908, La Documentation Française, Paris, janvier

Dupont Yves (dir.), 2007, Dictionnaire des risques, Armand Colin, Paris

Favier René et Granet-Abisset Anne-Marie (dir.), 2000, Histoire et Mémoire des Risques Naturels, CNRS, MSH-Alpes, Grenoble

Gallais Jean, 1994, Les tropiques, terres de risques et de violences, Armand Colin, Paris. Guilhou Xavier et Lagadec Patrick, 2002, La fin du risque zéro, Eyrolles, Paris.

Gwinner Chloé, 2012, « Traitement médiatique de la grippe H1N1 par deux quotidiens québécois : les journaux au service de la Direction », Les Cahiers du journalisme $n^{\circ} 24$, Université Laval, Québec, pp. 72-93.

Joffe Hélène, 2005, « De la perception à la représentation du risque : le rôle des médias », Hermès, La Revue, $n^{\circ}$ 41, /1, http://www.cairn.info/revue-hermes-la-revue-2005-1-page-121.

htm\#anchor_citation, consulté le 12 décembre 2015.

Macé Eric, 2006, Les imaginaires médiatiques. Une sociologie postcritique des Médias, Paris, Editions Amsterdam

Marchetti Dominique, 2010, Quand la santé devient médiatique. Les logiques de production de l'information dans la presse, Grenoble, Presses de l'Université de Grenoble 
Lesourd Michel, Amat-Roze Jeanne-Marie, 2003, Le risque : vulnérabilité et défis, Temps, Paris Libaert Thierry, 2010, La communication de crise (3e édition), Dunod, Paris

Mathien Michel, 1992, Les journalistes et le système médiatique, Hachette Supérieur, Paris

Métailié Jean-Paul et Bertrand Georges, 2006, Les mots de l'environnement, Presses universitaires du Mirail, Université de Toulouse-Le Mirail.

Minc Alain, 1993, Le Média-choc, Grasset \& Fasquelle, Paris.

Moriniaux Vincent (collectif), 2003, Les risques, collection «Questions de géographie », Éditions du Temps, Nantes.

Nau Jean-Yves, août 2014, Ebola : Ce ne sont pas les journalistes qui couvrent le mieux l'épidémie, Slate Afrique.

Ndiaye Adama, 2015, « Enjeux et risques de la mondialisation de l'information dans le tourisme sénégalais » in Pomel S. (dir.), Du risques en Afrique. Terrains et perspectives, Karthala-MSHA, Pessac, pp. 63-72

Ndiaye Adama, 2012, Communication, Tourisme et Développement durable : enjeux et risques au Sénégal, thèse de Université Michel de Montaigne Bordeaux 3

Percie du Sert Anne-Marie, 1999, Risque et contrôle du risque, Economica, Paris

Richter L. K. and Waugh W. L. Jr., 2003, « Terrorism and tourism as logical companions », in Colin Michael Hall, Dallen J. Timothy, David Timothy Duval (dir.), Safety and security in tourism :

relationships, management and marketing, Routledge, London

Veyret Yvette (dir.), 2003, Les risques, Dossiers des Images Économiques du Monde, SEDES, Paris.

\section{NOTES}

1. Ministère du Tourisme et des Transports aériens du Sénégal, rapport de synthèse du plan stratégique de développement durable du Tourisme 2014 / 2018, p. 4.

1. Ministère du Tourisme et des Transports aériens du Sénégal, rapport de synthèse du plan stratégique de développement durable du Tourisme 2014 / 2018, p. 4.

2.

3. Ibidem, p. 3.

4. Les mots de l'environnement,Jean-Paul Métailié et Georges Bertrand, Presses universitaires du Mirail, Université de Toulouse-Le Mirail, 2006, p. 100.

\section{RÉSUMÉS}

La dimension de plus en plus importante de l'économie touristique à l'échelle mondiale en fait un secteur éminemment stratégique. Ainsi, devant l'ampleur des risques d'aujourd'hui, leur caractère divers et multiforme (épidémie, terrorisme, catastrophes naturelles etc.), les politiques sont interpelés de tout bord. L'énigmatique cas de l'épidémie Ebola au Sénégal, démontre encore la vulnérabilité du tourisme, qui reste la proie du moindre risque ambiant. Mais la fulgurante 
révolution médiatique, certes est un atout dans l'acquisition de savoirs, dans la diffusion de l'information et le partage de connaissances, mais elle s'avère aussi une redoutable arme d'amplification de nouvelles alarmantes. L'actualité d'une mise en quarantaine de l'Afrique Occidentale par la communauté internationale en 2014 et l'impact du désastre économique occasionné - parce que les médias ont informé à outrance sur cette épidémie - a failli tuer un secteur névralgique de l'économie sénégalaise. Devant une telle situation, les médias deviennent un enjeu majeur dans la gestion de crise de cette nature. Telle est la réflexion que suscite cet article articulé autour du risque sanitaire, son amplification médiatique et ses impacts sur une économie touristique vulnérable par essence.

The growing importance of the tourism economy in the world makes it a highly strategic sector. Thus, given the magnitude of risk today and their diverse and multifaceted nature (epidemics, terrorism, natural disasters etc.) policies are arraigned on all sides. The enigmatic case of the Ebola epidemic in Senegal, discussed in the text, further demonstrates the vulnerability of tourism, which remains a prey at bay surrounding the slightest risk. But the media revolution, which certainly remains an asset in the acquisition of knowledge and dissemination of information, is also proving to be a formidable weapon of alarming news. The current quarantine of West Africa by the international community in 2014 and the impact of the economic disaster it caused and because the media informed about excessive Ebola outbreak, nearly killed tourism which is a key sector of the local economy. In such a situation, it is wise to reflect on the media challenge in managing crisis of this nature. Such is the thinking that creates this article centred on the health risk, its media amplifying and impacts on a tourist economy that is vulnerable by nature.

La dimensione del turismo, sempre più importante su scala mondiale, lo rende un settore altamente strategico per l'economia. Così, visto l'aumento dei rischi di oggi, a una loro diversa e moltepli cenature (epidemie, terrorismo, catastrofi naturali, ecc ), i politici sono chiamati in causa da più parti. Il misterioso caso di epidemia di Ebola in Senegal, trattato in questo testo, dimostra ancora una volta la vulnerabilità del turismo, che rimane una facile preda colpita anche da eventi di poca importanza. Ma l'importante rivoluzione mediatica,che è certamente un bene per l'acquisizione di conoscenze, diffusione di informazioni e condivisione delle conoscenze, si dimostra anche una pericolosa arma in grado di amplificare negativamente le informazioni. La notizia della messa in quarantena dell'Africa Occidentale da parte della comunità internazionale nel 2014 e il disastroso impatto economico che ha causato (perché i media hanno diffuso notizie in modo esagerato su questa epidemia di Ebola) hanno quasi ucciso un settore chiave dell'economia senegalese. Davanti ad una situazione simile i media diventano una complicanza nella gestione di una crisi di questo tipo. Tale è in effetti la riflessione che suscita questo articolo incentrato sui rischi sanitari, sulla loro amplificazione mediatica e sul loro impatto nell'economia turistica, vulnerabile per natura.

\section{INDEX}

Mots-clés : tourisme, risque, ebola, communication, médias.

Parole chiave : turismo, rischio, ebola, la comunicazione, media

Keywords : tourism, risks, ebola, media, communication 
AUTEURS

ADAMA NDIAYE

Université Sine Saloum Elhadj Ibrahima Niass (USSEIN)

MOUSSA MBOW

Institut Supérieur des Sciences de l'Information et de la Communication (ISSIC) 